\title{
Le transport vésiculaire des cellules eucaryotes est contrôlé par des GTP-ases
}

Des progrès remarquables ont été accomplis ces dernières années dans la compréhension des mécanismes moléculaires qui sous-tendent la formation, l'adressage et la fusion des vésicules de transport intracellulaire. De nombreuses protéines, tant structurales que régulatrices, impliquées dans ces processus ont été récemment identifiées. Une des découvertes majeures a été la mise en évidence, sur la membrane de plusieurs compartiments cellulaires, de protéines capables de fixer et d'hydrolyser le nucléotide GTP. Ces GTP-ases, dont la plupart appartiennent à la superfamille des protéines $\mathrm{p} 21^{\text {ras }}$, permettent à la cellule eucaryote de contrôler les différentes étapes du transport intracellulaire.

\section{Bruno Goud}

\section{Remerciements}

Je remercie Andrée Tixier-Vidal et Daniel Louvard pour leurs commentaires sur ce manuscrit.

\section{ADRESSE ET TIRÉS À PART}

B. Goud : directeur de recherche au Cnrs. Unité de génétique somatique, URA Cnrs 361, finte 1entenant les macromolécules trans-

de génétique somatique, URA Cnrs 361, contenant les macromolécules trans75724 Paris Cedex 15, France. portées (membranaires ou solubles e transport vésiculaire est une fonction essentielle qui permet d'acheminer d'un endroit à l'autre de la cellule eucaryote les macromolécules (protéines et certains lipides) synthétisées par la cellule (voie de biosynthèse/sécrétion) et celles provenant du milieu extracellulaire (voie d'endocytose). Pendant leur trajet, les macromolécules transportées traversent successivement plusieurs organelles ou compartiments intracellulaires. Le transport est un processus complexe, plus ou moins développé ou spécialisé suivant les types cellulaires. Chaque événement de transport entre deux compartiments peut cependant se décomposer en trois étapes relativement simples schématisées sur la figure 1: formation d'une vésicule dans la lumière) par bourgeonnement à partir du compartiment donneur, " adressage " (ou ciblage) de ces vésicules vers le compartiment accepteur et enfin fusion des vésicules avec la membrane de ce compartiment.

Le processus de transport est soumis à deux impératifs fondamentaux : il doit être unidirectionnel, pour permettre un transport vectoriel efficace des macromolécules, et étroitement réglé pour assurer le cheminement séquentiel des vésicules au travers des différents compartiments. Une série de travaux, initiés chez la levure Saccharomyces cerevisiae il y a quelques années, indiquent que des protéines capables de lier et d'hydrolyser le GTP (GTP-ases), présentes sur la membrane des compartiments cellulaires, permettent à la cellule de répondre à ces contraintes. Ces GTPases appartiennent à la superfamille 
des protéines ras (ou superfamille des "petites protéines $G$ ") dont elles constituent maintenant une branche distincte, la famille Sec4/Ypt1/rab. Très récemment, d'autres GTP-ases, comme le facteur d'ADP-ribosylation $A R F$ et des protéines $G$ hétérotrimériques ont été impliquées dans la régulation du transport et du trafic membranaire. Nous évoquerons ces résultats encore préliminaires à la fin de cette revue.

\section{Genèse de la famille de gènes SEC4/YPT1/rab}

SEC4 et YPT1 sont deux gènes de Saccharomyces cerevisiae (la levure de bière), un eucaryote unicellulaire qui a fourni un modèle remarquable pour l'étude des mécanismes moléculaires du transport depuis dix ans. Bien que cloné après YPT1, SEC4 a été le premier gène de la superfamille ras impliqué dans un processus de transport intracellulaire. La découverte de SEC4 remonte au début des années 1980 quand Novick et Schekman, à l'université de Berkeley (CA, USA), isolent des mutants thermosensibles de sécrétion chez Saccharomyces cerevisiae $[1,2]$. Chez la levure, la voie de biosynthèse/sécrétion des protéines est comparable à la voie de sécrétion constitutive (le transport a lieu sans stockage apparent des macromolécules à l'intérieur des compartiments) des cellules de mammifères. Ces mutants thermosensibles ont permis de caractériser une première série de gènes (on en connaît actuellement plus de 25) qui gouvernent le transport des protéines depuis leur translocation dans la lumière du réticulum endoplasmique jusqu'à leur acheminement vers la membrane plasmique et l'espace périplasmique. Le gène SEC4 fait partie des gènes dit tardifs : une mutation thermosensible dans SEC4 provoque une accumulation intracellulaire de vésicules dérivées de l'appareil de Golgi contenant les protéines membranaires ou sécrétées bloquées dans leur transit vers la membrane plasmique. En 1987, Salminen et Novick, à l'université Yale (CT, USA), clonent et séquencent le gène SEC4 et montrent qu'il code une protéine de poids moléculaire $23,5 \mathrm{kDa}$ possédant les domaines consensus impliqués dans la fixation et l'hydro$m / s n^{\circ} 4$, vol. 8 , avril 92 lyse du GTP des protéines ras (Sec4 possède $32 \%$ d'homologie avec $\mathrm{H}$ ras) [3]. Ces auteurs montrent en outre que la duplication du gène SEC4 permet d'abolir l'effet de mutations thermosensibles de trois autres gènes intervenant dans le transport entre l'appareil de Golgi et la membrane plasmique, ce qui indique que SEC4 est un gène régulateur important de cette étape du transport. Par la suite, la purification de la protéine Sec4 confirmera que Sec4 lie avec une très haute affinité le GTP (et le
GDP) et possède une faible activité GTP-asique intrinsèque, deux caractéristiques des protéines de la superfamille ras (voir plus bas) [4].

Le gène YPT1 code une protéine de $23 \mathrm{kDa}$ apparentée à ras et possédant $45 \%$ d'homologie avec Sec4. Le gène YPT1 a été trouvé un peu par hasard par l'équipe de Dieter Gallwitz (Allemagne) comme cadre de lecture ouvert entre les gènes codant pour la tubuline et l'actine de Saccharomyces cerevisiae [5]. Ces auteurs ont proposé que YPT1 intervenait dans

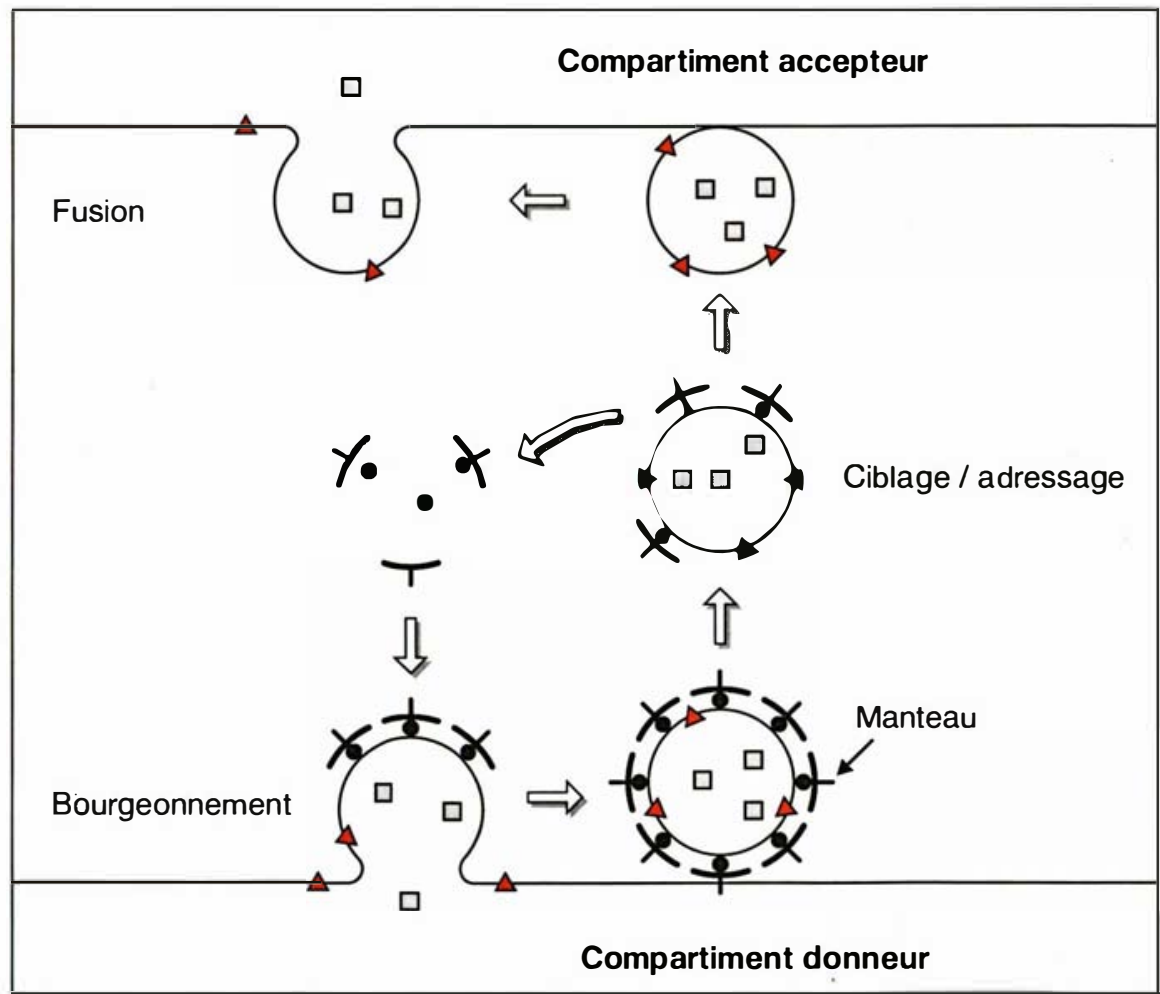

Figure 1. Les trois étapes du transport entre deux compartiments. La vésicule de transport contenant les macromolécules transportées - membranaires (triangles rouges) ou solubles (carrés gris) - dans la lumière se forme à partir du compartiment donneur. Dans plusieurs étapes du transport, on a montré que ce processus implique le recrutement à partir du cytosol de protéines (- et $\downarrow$ ) qui vont former un manteau (coat) recouvrant la vésicule de transport. Plusieurs protéines de ce manteau sont maintenant identifiées. Dans la voie d'endocytose (formation des vésicules à partir de la membrane plasmiquel, le manteau est composé de clathrine et d'une famille de protéines appelées adaptines. Dans le transport intra-golgien (voie de biosynthèse/sécrétion), le manteau est composé d'une molécule clathrin-like non encore identifiée et $d^{\prime} u n e$ protéine récemment identifiée ( $\beta$-COP) qui possède des homologies de séquence avec les adaptines. Le manteau se dissocie probablement lorsque la vésicule est formée. Après bourgeonnement, la vésicule est ciblée vers le compartiment accepteur. Ce processus, encore mal connu, fait intervenir les éléments du cytosquelette, en particulier le réseau microtubulaire chez les cellules de mammifères. La vésicule s'ancre ensuite sur la membrane du compartiment accepteur. Une machinerie protéique complexe se met alors en place (impliquant aussi le recrutement de protéines cytosoliques) qui aboutit au processus de fusion des deux membranes. 


\section{RÉFÉRENCES}

1. Novick P, Ficld C, Schekman R. Identification of 23 complementation groups required for post-translational events in the ycast secretory pathway. Cell $1980 ; 21$ : 205-15.

2. Novick P, Ferro S, Schekman R. Order of events in the yeast secretory pathway. Cell 1981; $25: 461-9$.

3. Salminen A, Novick P. A ras-like protein is required for a post-Golgi cvent in ycast sccretion. Cell $1987 ; 47$ : 527-38.

4. Kabcencll AK, Goud B, Northup J, Novick P. Binding and hydrolysis of guanine nucleotides by Scc4p, a yeast protein involved in the regulation of vesicular traffic. $J$ Biol Chem 1990; 265 : 9366-72.

5. Gallwitz D, Donath C, Sander C. A ycast gene cncoding a protcin homologous to the human c-has/bas proto-oncogene product. Nature 1983 ; 306 : 704-7.

6. Segev N, Mulholland J, Botstein D. The ycast GTP-binding YPT1 protein and a mammalian counterpart are associated with the secretion machincry. Cell $1988 ; 52$ : 915-24.

7. Bacon $\mathrm{R}$, Salminen A, Ruohola $\mathrm{H}$ Novick P, Ferro-Novick S. The GTPbinding protcin YPT1 is required for transport in vitro: the Golgi apparatus is defective in YPT1 mutants. J Cell Biol 1989 ; 109 : 1015-22.

8. Baker D, Wucstchube L, Schckman R, Botstcin D, Scgev N. GTP-binding Ypt1 protein and $\mathrm{Ca}^{2}+$ function independently in a cell-frec protcin transport reaction. Proc Natl Acad Sci USA 1990; 87 : 355-9.

9. Scgev N. Mediation of the attachment or fusion step in vesicular transport by the GTP-binding Ypt1 protein. Science 1991 ; 252: 1553-6.

10. Touchot $\mathrm{N}$, Chardin $\mathrm{P}$, Tavitian $\mathrm{A}$. Four additional members of the ras gene superfamily isolated by an oligonucleotide strategy : molecular cloning of YPT-related cDNAs from a rat brain library. Proc Natl Acad Sci USA 1987 ; 84: 8210-4.

11. Zahraoui A, Touchot N, Chardin P, Tavitian A. The human rab genes encode a family of GTP-binding proteins related to ycast YPT1 and SEC4 products involved in secretion. J Biol Chem 1989; 264 : 12394-401.

12. Chavrier $P$, Vingron $M$, Sander $C$, Simons K, Zerial M. Molecular cloning of YPT1/SEC4-rclated cDNAs from an cpithelial cell linc. Mol Cell Biol 1990; 10 : 6578-85.

13. Chavrier P, Simons K, Zerial M. The complexity of the rab and rho GTP-binding protein family revealed by a PCR cloning approach. Oncogene 1992 (sous pressc).

14. Haubruck H, Prange R, Vorgias C, Gallwitz D. The ras-related mousc ypt1 protein can functionally replace the YPT1 gene product in yeast. $E M B O J 1989 ; 8$ : 1427-32. l'organisation du réseau microtubulaire, puis dans la régulation du taux de calcium intracellulaire. Mais, à la suite des travaux initiés sur SEC4, plusieurs équipes ont montré que le principal phénotype des mutants thermosensibles de YPT1 était une altération du processus de transport des protéines entre le réticulum endoplasmique et l'appareil de Golgi et ont proposé qu'YPT1 jouait un rôle analogue à SEC4 dans une étape plus précoce de la voie de sécrétion [6-9]. Les premiers gènes rab ont été caractérisés par l'équipe d'Armand Tavitian (Paris, France) qui s'intéressait à l'isolement de nouveaux gènes de la famille ras chez les mammifères. En criblant une banque d'ADNc de cerveau de rat par un oligonucléotide correspondant à un motif du site nucléotidique des protéines ras (site G3 sur la figure 2), ces auteurs ont isolé, en 1987, quatre gènes possédant 35 à $75 \%$ d'homologie avec SEC4/YPT1. L'analyse de leur séquence montrant qu'ils définissaient probablement une sous-famille de ras, ils ont nommé ceux-ci rab1, rab2, rab3 et rab4 (rab signifie ras genes from rat brain) [10]. Par la suite, le même groupe isolera leurs homologues chez l'homme, ainsi que quatre nouveaux gènes rab (rab1B, $r a b 3 B, r a b 5$ et rab6) [11]. Grâce aux travaux de l'équipe de Marino Zerial à l'EMBL (Heidelberg, Allemagne), la famille des gènes rab s'est récemment enrichie de nouveaux éléments, trouvés en particulier par PCR (polymerase chain reaction) $[12,13]$. Cette famille comprendrait aujourd'hui entre 20 et 30 membres !

La famille des gènes rab est d'abord définie par une homologie de séquence avec SEC4/YPT1. Toutefois, de nombreux arguments permettent de penser que ces gènes sont effectivement impliqués dans les processus de transport intracellulaire des cellules de mammiferes (voir plus bas). Le gène rab1A (75 \% d'homologie entre rab1A et $Y p t 1)$ peut d'autre part remplacer fonctionnellement YPT1 chez la levure, indiquant qu'il s'agit de son homologue chez les cellules de mammiferes [14]. Les gènes rab caractérisés jusqu'à présent semblent quasiment conservés chez les différentes espèces de mammifères. On a récemment trouvé pour certains d'entre eux des homologues chez les levures Saccharomyces cerevisiae et Schizosaccharomyces pombe, et, très probablement, la majorité des gènes rab est présente chez tous les eucaryotes. On connaît encore mal leur expression dans les différents types cellulaires, mais la majorité des protéines rab identifiées jusqu'à présent semblent ubiquitaires. Une de ces protéines, rab3A, n'est cependant exprimée que dans les cellules neuronales et neuroendocrines [15], et il est probable que dans un proche avenir d'autres protéines rab spécifiques de certains tissus ou types cellulaires seront découvertes. Par ailleurs, l'expression de certaines protéines rab semble augmenter au cours du processus de différenciation de certaines cellules, par exemple les cellules de la lignée monocyte/macrophage [16].

\section{Structure des protéines Sec4/Ypt 1/rab et principales caractéristiques biochimiques}

Les protéines Sec4/Ypt1/rab constituent à l'heure actuelle une branche distincte de la superfamille des protéines ras, les deux autres branches principales étant formées par les protéines $\mathrm{H}$-ras, K-ras et $\mathrm{N}$-ras, et leurs parents proches (rap, ral...) ainsi que par les protéines rho et leurs parents proches (rac...). Cette superfamille s'est considérablement agrandie ces dernières années puisqu'elle comprend maintenant environ une centaine de membres impliqués dans un grand nombre de fonctions cellulaires. Les protéines de la superfamille ras possèdent entre elles au moins $30 \%$ d'homologie. Elles sont toutes construites sur le même modèle représenté sur la figure 2. Ce dernier s'articule autour de domaines très conservés (représentés en noir sur la figure 2) qui sont impliqués dans la fixation et l'hydrolyse du GTP. L'analyse cristallographique de H-ras a montré que ces domaines forment, après repliement de la protéine native, une poche dans laquelle s'insèrent les nucléotides GDP ou GTP. Des domaines proches (à l'exception de G5) sont retrouvés dans toutes les GTP-ases connues (sous-unités $\alpha$ des protéines $G$ hétérotrimériques, facteurs d'élongation, protéines ARF...). Plusieurs 


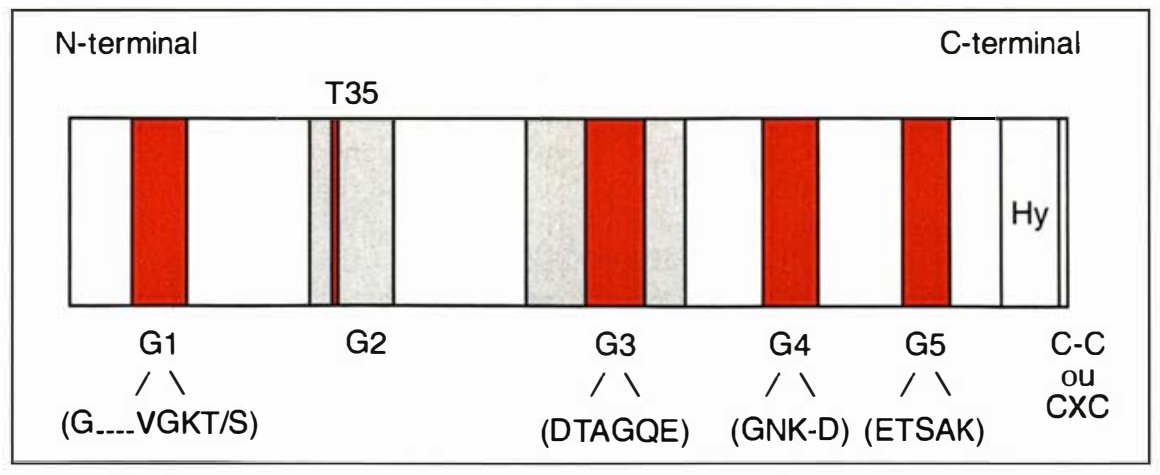

Figure 2. Structure des protéines Sec4/Ypt1/rab. Les domaines marqués en rouge sont ceux impliqués dans la fixation et l'hydrolyse du GTP et qui sont conservés chez tous les membres de la superfamille ras. G1 et G3 interagissent avec les résidus phosphates; G4 et G5, avec le noyau guanosine du GTP et du GDP. Les domaines en grisé ont de fortes homologies de séquence chez tous les membres de la famille Sec4/Ypt1/rab. G2 correspond à la partie effectrice des protéines p21ras lla thréonine en position 35 de ce domaine est conservée chez tous les membres de la superfamille). Hy représente la région hypervariable impliquée dans l'adressage de ces protéines vers les différents compartiments cellulaires.

excellentes revues ont été récemment consacrées à l'analyse structurale des sites nucléotidiques des GTPases $[17,18]$. Nous montrons sur la figure 2 les motifs consensus des sites nucléotidiques des protéines de la superfamille ras.

Plusieurs régions (représentées en gris sur la figure 2) apparaissent plus conservées chez les protéines Sec4/Ypt1/rab que chez les autres membres de la superfamille ras. Ces régions incluent le domaine ( $\mathrm{G} 2$ sur la figure 2) compris entre les acides aminés 32-42 (numérotés en alignant les séquences de Sec4/Ypt1/rab sur celles de H-ras) et qui correspond à la région dite effectrice de $\mathrm{p} 21^{\text {ras }}$ (effector loop). Ce domaine effecteur interagit en particulier avec les protéines qui stimulent l'activité d'hydrolyse du GTP (protéine GAP pour GTP-ase activating protein) des protéines ras. La conservation de cette région dans la famille Sec4/Ypt1/rab suggère l'existence d'effecteurs communs ou proches pour toutes les protéines de cette famille et différents de ceux des autres membres de la superfamille ras. On a pu montrer, par exemple, que des peptides synthétiques mimant cette région bloquent plusieurs étapes de transport in vitro [19].

L'extrémité carboxy-terminale est aussi caractéristique des protéines Sec4/Ypt1/rab. Ces protéines se ter$m / s n^{\circ} 4$, vol. 8, avril 92 moléculaire compris entre 20 et $26 \mathrm{kDa}$ (c'est pourquoi on les appelle aussi petites protéines $\mathrm{G}$ par comparaison aux "grandes " protéincs $G$ hétérotrimériques dont les sous-unités $\alpha$ ont un poids moléculaire d'environ $40 \mathrm{kDa}$ ). Elles ont une très haute affinité pour le GTP et le GDP ( $\mathrm{K}_{\mathrm{d}}$ compris entre $10^{-11}$ et $10^{-7} \mathrm{M}$ ) et une activité intrinsèque d'hydrolyse du GTP en GDP très faible. Le passage de la forme liée au GTP à celle liée au GDP (et vice versa) s'accompagne, par ailleurs, d'un changement conformationnel de la protéine [17, 18]. Il faut cependant souligner que seule la biochimie de $\mathrm{H}$-ras a été étudiée en détail. Il est très probable que des différences existent d'une protéine à l'autre ou d'une famille de protéines à l'autre. L'étude biochimique de Sec4 a, par exemple, montré que cette protéine a plus d'affinité pour le GTP que pour le GDP, contrairement à $\mathrm{H}$-ras qui a la même affinité pour les deux nucléotides [4]. par les motifs CC ou CXC $(\mathrm{C}=$ cystéine et $\mathrm{X}$, n'importe quel acide aminé). Cette extrémité diffère de celle de la majorité des autres protéines ras et apparentées qui se terminent par CAAX (A : acide aminé aliphatique). La région carboxyterminale des protéines de la superfamille ras est impliquée dans l'attachement de ces protéines aux membranes, un processus crucial pour leur fonction. Les formes précurseurs sont en effet cytosoliques et subissent une série de modifications posttraductionnelles qui leur permet de s'insérer dans la bicouche lipidique des membranes. Cette maturation est maintenant en partie élucidée pour les protéines ras grâce à des travaux récents, en particulier ceux du groupe de Chris Marshall (Angleterre) : elle consiste en une isoprénylation, protéolyse et carboxy-méthylation qui laissent la cystéine du motif CAAX en position C-terminale farnésylée (isoprényl à 15 carbones) et méthylestérifiée [20]. Dans le cas des protéines Sec4/Ypt1/rab, les remaniements de l'extrémité C-terminale sont encore mal connus. On sait toutefois que la cystéine ou les cystéines terminales ne sont pas farnésylées, mais géranyl-géranylées (isoprényl à 20 carbones) [21].

Les protéines de la superfamille ras sont monomériques et ont un poids

\section{Localisation intracellulaire des protéines Sec4/Ypt 1/rab}

On ne connaît la localisation intracellulaire que d'une dizaine de protéines de la famille Sec4/Ypt1/rab. Mais on a trouvé, dès à présent, au moins une de ces protéines sur les principaux compartiments intracellulaires impliqués dans le transport des macromolécules. La première à avoir été localisée à l'aide d'anticorps spécifiques est Sec4, qui est présente sur la face cytoplasmique des vésicules de sécrétion issues de l'appareil de Golgi et sur la face interne de la membrane plasmique [22]. Toujours chez Saccharomyces cerevisiae, on a pu montrer que Ypt1 est associée à l'appareil de Golgi [6]. La figure 3, p. 331 décrit la localisation des protéines rab étudiée en microscopie optique ou électronique dans les cellules de mammiferes : dans la voie de biosynthèse/sécrétion, au moins trois protéines (rab1A, rab1B, rab2) sont associées avec l'interface réticulum endoplasmiqueface cis de l'appareil de Golgi, en particulier avec un compartiment dit intermédiaire (ou salvage compartment) très étudié actuellement et qui joue un rôle crucial dans le transport vers 


\section{RÉFÉRENCES}

15. Olofsson B, Chardin P, Touchot $N$, Zahraoui A, Tavitian A. Expression of the ras-related rabA, rho and rab genes in adult mouse tissues. Oncogene $1988 ; 3$ : 231-4. 16. Maridonneau-Parini I, Yang C, Bornens $M$, Goud $B$. Increase in the expres sion of a family of small GTP-binding proteins, rab proteins, during induced phagocyte differentiation. J Clin lnvest 1991; 87 : 901-7.

17. Bourne H, Sanders D, McCormick F The GTPase superfamily: a conserved switch for diverse cell functions. Nature $1990 ; 348: 125-32$.

18. Bourne H, Sanders D, McCormick F The GTPase superfamily : conserved structure and molecular mechanism. Nature $1991 ; 349: 117-27$.

19. Plutner $H$, Schwaninger $R$, Pind $S$ Balch W. Synthetic peptides of the Rab effector domain inhibit vesicular transport through the secretory pathway. EMBO J 1990 ; 9 : 2375-83.

20. Hancock J, Magee A, Childs J, Marshall C. All ras proteins are polyisoprenylated but only some are palmitoylated. Cell 1989 ; 57 : 1167-77.

21. Khosravi-Far R, Lutz R, Cox A, el al. Isoprenoid modification of rab proteins terminating in CC or CXC motifs. Proc Natl Acad Sci USA 1991; 88 : 6264-8.

22. Goud B, Salminen A, Walworth $\mathrm{N}$, Novick P. A GTP-binding protein required for secretion rapidly associates with secre tory vesicles and the plasma membrane in yeast. Cell $1988 ; 53$ : 753-68.

23. Chavrier $P$, Parton $R$ Hauri HP Simons K, Zerial M. Localization of lowmolecular weight GTP-binding proteins to exocytic and endocytic compartments. Cell $1990 ; 62: 317-29$.

24. Plutner H, Cox A, Pind S, et al. Rab1b regulates vesicular transport between the endoplasmic reticulum and successive Golgi compartments. J Cell Biol 1991; 115. 31-43.

25. Goud B, Zahraoui A, Tavitian A, Saraste J. Small GTP-binding protein associated with Golgi cisternae. Nature 1990 ; 345: 553-6.

26. Jasmin C, Goud B, Camus G, Cartaud J. Association of rab6p with assymetrically distributed post-Golgi vesicles in a polarized cell. Neuroscience 1992 (sous presse)

27. Fisher von Mollard G, Mignery G, Baumert $\mathrm{M}$, et al. Rab3 is a small GTP binding protein exclusively localized to synaptic vesicles. Proc Natl Acad Sci USA $1990 ; 87$ : 1988-92.

28. Darchen F, Zahraoui A, Hammel F, Monteils M-P, Tavitian A, Scherman D. Association of the GTP-binding protein Rab3A with bovine adrenal chromaffin granules. Proc Natl Acad Sci USA 1990 ; 87 5692-6. le Golgi des protéines issues du réticulum endoplasmique $([23,24]$; B. Goud et J. Saraste, résultats non publiés). Rab6 est associée dans tous les types cellulaires aux dernières citernes de l'appareil de Golgi et au trans-Golgi network (TGN), une région impliquée dans le tri des protéines à la sortie de l'appareil de Golgi ([25] ; C. Antony et al., résultats non publiés). Dans les neurones, rab6 est aussi associée aux vésicules synaptiques (A. Tixier-Vidal, résultats non publiés) et, dans l'organe électrique de Torpedo marmorata, aux vésicules post-golgiennes [26]. Rab8 semble associée aux vésicules qui acheminent les protéines vers la membrane basolatérale des épithéliums (K. Simons, communication personnelle). Dans les neurones, rab3A est présente sur les vésicules synaptiques [27] ; cette protéine a aussi été trouvée sur la membrane des granules des cellules chromaffines [28]. Trois protéines rab sont associées aux compartiments de la voie d'endocytose: il s'agit de rab4 et rab5 (endosomes précoces), ainsi que de rab7, trouvée sur les endosomes tardifs $[23,29]$.

Comme la famille compte au minimum 25 membres déjà identifiés, il est probable que plusieurs protéines rab sont présentes dans les mêmes compartiments intracellulaires (comme c'est déjà le cas pour rab4 et rab5), ce qui ne manquera pas de compliquer l'étude de la fonction respective de chacune de ces protéines. Il est possible aussi que certaines protéines rab soient associées à des processus de transport spécifiques ou particulièrement développés dans certains types cellulaires.

Une caractéristique des protéines Sec4/Ypt1/rab est qu'elles existent à l'état d'équilibre dans la cellule sous une forme cytoplasmique [23, 25, 29]. Cela suggère qu'elles peuvent s'associer et se dissocier facilement des membranes par des mécanismes encore pratiquement inconnus. On a pu cependant montrer que, dans les cellules en mitose, l'association/dissociation des membranes des protéines rab1 A et rab4 est couplée à un événement de phosphorylation contrôlée par la kinase p34 cdc2 [30].

Un point capital et non encore élucidé est de savoir comment chaque protéine rab est ciblée vers tel ou tel compartiment intracellulaire. Une étude du groupe de Marino Zerial a montré récemment que les protéines rab contenaient un signal de ciblage situé dans la région hyperviable près de l'extrémité C-terminale (voir figure 2) [31]. Une hypothèse probable est qu'il existe des " récepteurs" spécifiques pour ces séquences sur chaque compartiment intracellulaire.

\section{Fonction et mode d'action des protéines Sec4/Ypt1/rab}

S'il est probable que tous les membres de cette famille de protéines sont impliqués dans des phénomènes de transport, on ne dispose à l'heure actuelle de preuves directes que pour Sec4 et Ypt 1. Ces preuves découlent essentiellement de l'étude génétique de la fonction de ces deux protéines chez Saccharomyces cerevisiae. En ce qui concerne Sec4, plusieurs mutations ont été introduites dans la protéine dont on a analysé l'effet sur le processus de sécrétion. Quand la protéine est bloquée dans sa conformation active (liée au GTP), comme par exemple dans la mutation $\mathrm{N}^{133} \rightarrow \mathrm{Ile}^{133}$ (remplacement d'une asparagine par une isoleucine en position 133) qui correspond à une mutation transformante de H-ras, on observe un arrêt de la sécrétion et une accumulation intracellulaire de vésicules, c'est-à-dire un phénotype identique à celui des mutants thermosensibles de SEC4 [32]. L'ensemble de ces études a permis d'établir que Sec4 contrôle le ciblage des vésicules de transport post-golgiennes vers la membrane plasmique.

Ypt1p joue un rôle similaire à celui de Sec4p dans le ciblage des vésicules de transport contenant les protéines quittant le réticulum endoplasmique vers l'appareil de Golgi. Le rôle de Ypt1 a pu être aussi étudié in vitro puisqu'on a pu reconstituer dans des systèmes acellulaires le processus de transport entre le réticulum endoplasmique et l'appareil de Golgi [6-9]. Le rôle exact des protéines rab est encore inconnu. On sait cependant, grâce aux expériences de reconstitution du transport intracellulaire in vitro, que les analogues non hydrolysables du GTP, comme le GTP-yS, bloquent de façon irréversible prati- 
quement toutes les étapes du transport des protéines dans les cellules de mammifères (pour revue voir [33]). Les cibles du GTP- $\gamma \mathrm{S}$ sont probablement, dans la plupart des cas, les protéines rab. La protéine rab la mieux connue d'un point de vue fonctionnel est rab5, présente sur les endosomes précoces. Les groupes de Marino Zerial et Jean Gruenberg ont montré que des anticorps dirigés contre rab5 bloquent in vitro la fusion des endosomes entre eux et qu'un excès de rab5 stimule au contraire ce processus [34]. Des résultats non encore publiés (M. Zerial, communication personnelle) indiquent en outre que la surexpression transitoire in vivo de la protéine rab5 augmente la vitesse d'endocytose des cellules et accroît la taille des endosomes alors que la surexpression de la protéine mutante rab5-Ile $\mathrm{e}^{133}$ conduit au contraire à une fragmentation des endosomes et inhibe l'endocytose. Rab5 contrôlerait donc une des premières étapes du

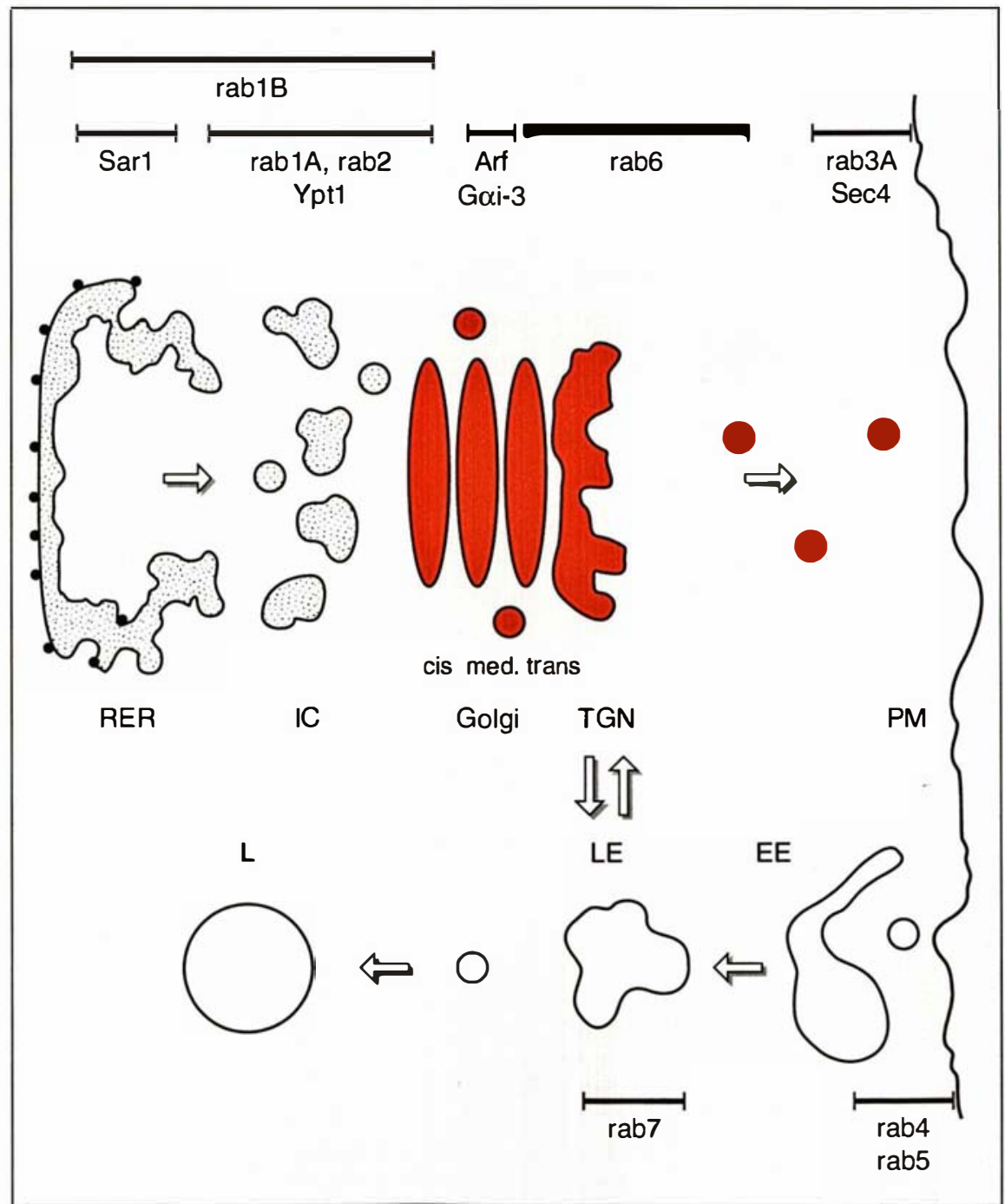

Figure 3. Localisation intracellulaire des GTP-ases. Des GTP-ases sont présentes sur la membrane des vésicules et des compartiments impliqués dans les voies de biosynthèse/sécrétion et d'endocytose. Voie de biosynthèse/sécrétion : RER : réticulum endoplasmique rugueux ; IC : compartiment intermédiaire ; G: Golgi; TGN : trans-Golgi network; PM : membrane plasmique. Voie d'endocytose: EE: endosomes précoces; $L E$ : endosomes tardifs; $L$ : lysosomes.

$\mathrm{m} / \mathrm{s} n^{\circ} 4$, vol. 8 , avril 92 processus d'endocytose. Située comme rab5 sur les endosomes précoces, rab4 interviendrait dans le processus de recyclage des protéines (récepteurs) endocytées (P. Van der Sluijs, communication personnelle). On possède aussi des informations sur la fonction de rab3A. Cette protéine serait impliquée dans l'" adressage " et/ou la fusion des vésicules synaptiques avec la membrane plasmique au niveau des synaptosomes [35]. Rab1B, un des homologues de Ypt1 chez les mammifères, intervient dans le ciblage des vésicules quittant le réticulum endoplasmique vers le cis-Golgi et dans le transport intra-golgien [24].

Comme pour toutes les GTP-ases, c'est le changement conformationnel entre la forme liée au GDP et celle liée au GTP qui sous-tend la fonction des protéines Sec4/Ypt1/rab. Pour les protéines $\mathrm{G}$ hétérotrimériques, ce changement de conformation permet la transduction d'un signal entre un récepteur membranaire activé et un effecteur qui va engendrer une cascade de messagers secondaires. Dans le cas du transport intracellulaire, le changement conformationnel permettrait à la GTP-ase d'osciller entre une forme cytosolique et une forme membranaire. Chaque phase du transport serait ainsi couplée au cycle d'une GTP-ase spécifique de cette étape [36]. Nous illustrons ce modèle cyclique sur la figure 4, p. 332 pour une GTP-ase impliquée dans un événement d'" adressage " (par exemple Sec4). Dans cet événement de transport, la vésicule migre vers sa membrane cible où elle s'ancre avant de fusionner. La cellule doit contrôler - que le ciblage de la vésicule est correct, c'est-à-dire que la vésicule va interagir avec le "bon" compartiment accepteur. C'est le rôle de la GTP-ase : sous sa forme inactive (liée au GDP), la GTP-ase est cytosolique ; elle s'active (remplacement du GDP par le GTP) en se fixant à la membrane de la vésicule. Elle reconnaîtrait ensuite sur la membrane acceptrice une protéine GAP qui stimulerait l'hydrolyse du GTP en GDP. Revenue sous sa forme GDP, la GTP-ase se dissocierait alors de la membrane de la vésicule et redeviendrait cytosolique. L'hydrolyse du GTP est donc conditionnelle, c'est-à- 


\section{RÉFÉRENCES}

29. Van der Sluijs $P$, Hull M, Zahraoui A Tavitian A, Goud B, Mellman I. The small GTP-binding protcin rab4 is associated with carly endosomes. Proc Natl Acad Sci USA $1991 ; 88: 6313-7$.

30. Bailly E, McCaffrey M, Touchot N,et al. Phosphorylation of two small GTPbinding proteins of the rab family by p34cdlc2. Nature $1991 ; 350$ : 715-8. 31. Chavrier P, Gorvel JP, Stelzer E, Simons K, Gruenberg J, Zerial M. Hypervariable C-terminal domain acts as a targeting signal. Nature $1991 ; 353: 769-72$. 32. Walworth N, Goud B, Kabcencll A, Novick P. Mutational analysis of SEC4 suggests a cyclical mechanism for the regulation of vesicular traffic. $E M B O J 1989 ; 8$ 1685-93.

33. Goud B, McCaffrey M. Small GTPbinding proteins and their role in transport. Curr Op Cell Biol 1991 ; 3 : 626-33.

34. Gorvel JP, Chavrier P, Zerial M, Gruenberg J. Rab5 controls carly endosome fusion in vitro. Cell 1991; $64: 915-25$

35. Fischer von Mollard G, Südhof $T$, Jahn R. A small GTP-binding protein dissociates from synaptic vesicles during exocytosis. Nature 1991 ; 349 : 79-82.

36. Bourne H. Do GTP-ases direct membranc traffic in secretion? Cell $1988 ; 53$ : 669-71.

37. Sasaki T, Kikuchi A, Araki S, Hata $Y$, Isomura $M$, Kuroda S, Takai Y. Purification and characterization from bovine brain cytosol of a protein that inhibits the dissociation of GDP from and the subsequent binding of GTP to smg p25A, a ras p21-like GTP-binding protein. J Biol Chem 1990 ; 265: 2333-7.

38. Stcarns $T$, Willingham $M$, Botstein $D$, Kahn R. ADP-ribosylation factor is functionally and physically associated with the Golgi complex. Proc Natl Acad Sci USA $1990 ; 87: 1238-42$.

39. Serafini T, Orci L, Amherd M, Brunner M, Kahn R, Rothman J. ADPribosylation factor is a subunit of the coat of Golgi-derived COP-coated vesicles : a novel role for a GTP-binding protcin. Cell 1991 ; 67 : 239-53.

40. Nakano A, Muramatsu M. A novel GTP-binding protein, Sarlp, is involved in transport from the endoplasmic reticulum to the Golgi apparatus. J Cell Biol $1989 ; 109$ : 2677-91.

41. D'Enfert C, Wucstchube L, Lila T, Schekman R. Sec 12p-dependent membranc binding of the small GTP-binding protein Sarlp promotes formation of transport vesicles from the ER. J Cell Biol 1991; 114 663-70.

42. Stow J, Bruno de Almeida J, Narula N, Holtzman E, Ercolani L, Ausicllo D. A heterotrimeric $G$ protein, $G \alpha_{1-3}$, on Golgi membranes regulates the secretion of a heparan sulfate protcoglycan in LLC-PK 1 cpithclial cells. J Cell Biol 1991; 114: 1113-24. dire qu'elle n'a lieu que si le ciblage d'adressage est correct. Si celui-ci n'est pas correct, l'hydrolyse du GTP ne peut se produire et le processus de transport est interrompu. L'hypothèse la plus simple est que GAP soit une des protéines participant à l'ancrage de la vésicule sur sa membrane cible. Toutefois, ce point fondamental du modèle n'a pas pu encore être vérifié expérimentalement. On ne sait pas non plus si une GTP-ase impliquée dans l' "adressage " intervient aussi dans la fusion proprement dite. L'hydrolyse du GTP pourrait en effet initier une série d'événements aboutissant à la fusion de la vésicule avec la membrane cible. Une autre hypothèse est qu'une autre GTP-ase contrôlant directement la machinerie de fusion prendrait le relais.

On peut imaginer le même type de modèle cyclique pour une GTP-ase contrôlant la formation d'une vésicule de transport à partir d'un compartiment donneur. Dans ce cas, l'hydrolyse du GTP a lieu quand la machincrie permettant le bourgeonnement de la vésicule est correctement assemblée. Bien que formellement non démontré, plusieurs éléments sont en faveur du modèle cyclique. L'un des plus convaincants est que les protéines Sec4/Ypt1/rab existent dans la cellule sous une forme cytoplasmique et une forme membranaire, à la différence de la majorité des autres protéines de la superfamille ras qui sont essentiellement membranaires. On a pu aussi montrer que rab3A se dissociait de la membrane des vésicules synaptiques après fusion de celle-ci avec la membrane des synaptosomes [35].

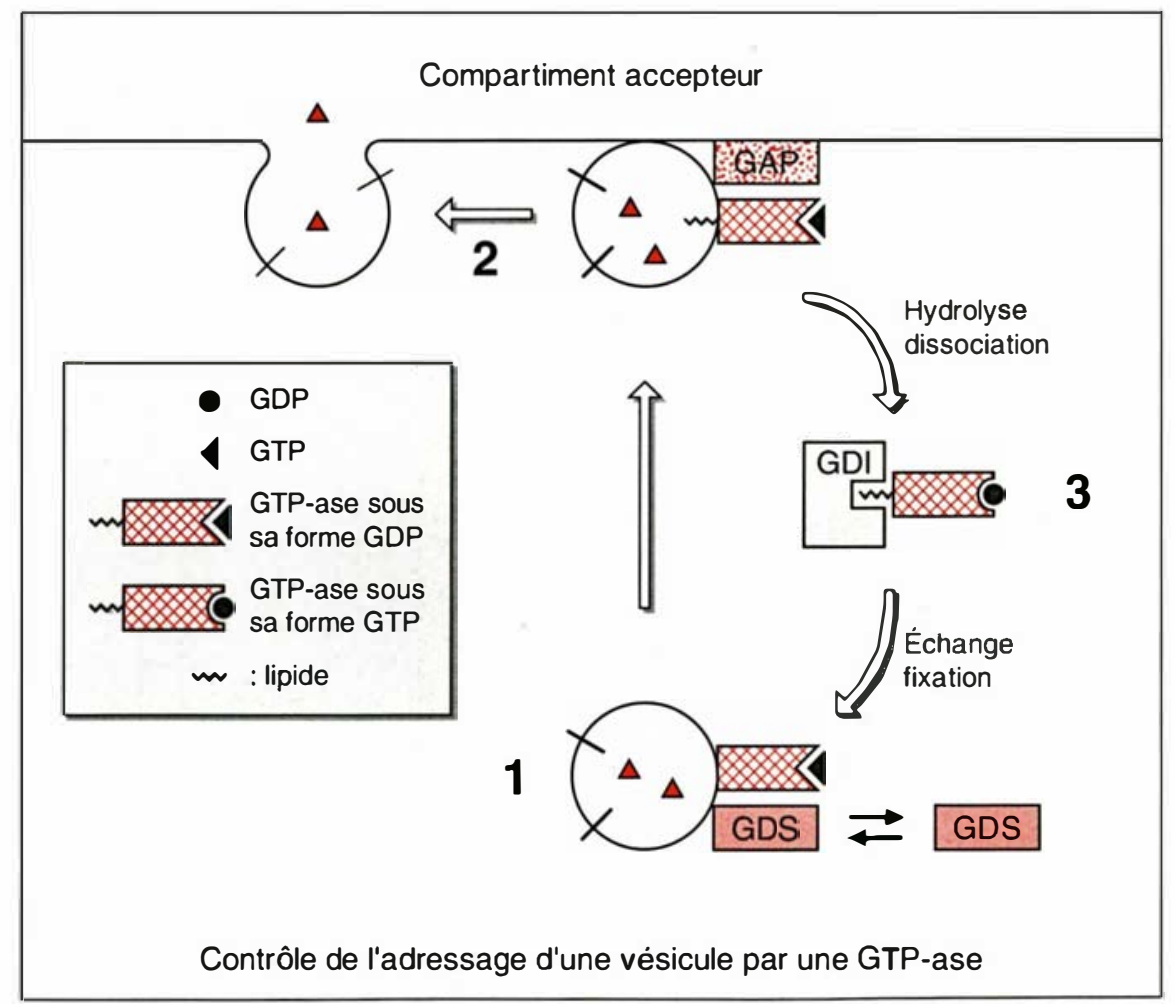

Figure 4. Contrôle de l' " adressage " d'une vésicule de transport par une GTP-ase. L'événement d'adressage de la vésicule de transport vers sa membrane cible est couplé au cycle d'une GTP-ase spécifique de cette étape. En (1), la vésicule lie, à sa surface, une petite protéine $G$ sous sa forme inactive, associée au GDP. En présence du facteur d'échange GDS, le GDP est remplacé par le GTP, activant la protéine G. En (2), elle interagit avec une protéine GAP qui provoque l'hydrolyse du GTP en GDP. En (3), la protéine $G$ est cytoplasmique, maintenue sous sa forme inactive, liée au GDP, par la protéine GDI, facteur inhibant la dissociation ; GAP : GTP-ase activating protein ; GDI : GDP dissociation inhibitor ; GDS : GDP dissociation stimulator (protéine d'échange). 
Comme pour toutes les GTP-ases, le changement conformationnel entre les formes GDP et GTP des protéines Sec4/Ypt1/rab est contrôlé par d'autres protéines. Elles incluent les facteurs d'échange qui favorisent le remplacement du GDP par le GTP (appelés aussi GDS pour GDP dissociation stimulator) et bien sûr les protéines GAP qui stimulent l'hydrolyse du GTP fixé $\left(\mathrm{m} / \mathrm{s} n^{\circ}\right.$ 7, vol. 6, p. 703 et $n^{\circ} 1$, vol. 8, p. 81). Ces protéines commencent à être relativement connues dans le cas de ras. En ce qui concerne la famille Sec4/Ypt1/rab, on ne sait encore pratiquement rien des protéines qui modulent le changement conformationnel. Le groupe de Yoshimi Takai au Japon a cependant récemment caractérisé une protéine appelée GDI qui inhibe la dissociation du GDP de rab3A (et donc la fixation du GTP) (GDI pour GDP dissociation inhibitory protein). GDI empêche aussi la fixation de rab3A sur les membranes et favorise la dissociation de la forme membranaire de rab3A [37]. GDI jouerait donc un rôle fondamental dans le modèle cyclique (nous faisons figurer GDI dans le modèle présenté sur la figure 4). Les protéines d'échange (GDS) n'ont pas été caractérisées. Une hypothèse séduisante est cependant que l'échange des nucléotides est couplé au processus de fixation des GTP-ases à leur membrane cible. Dans cette hypothèse, GDS pourrait en fait servir de récepteur membranaire pour les GTP-ases (voir figure 4).

\section{D'autres GTP-ases dans le transport?}

Des travaux très récents indiquent que d'autres GTP-ases n'appartenant pas à la famille Sec4/Ypt1/rab semblent jouer aussi un rôle important dans le transport intracellulaire. L'une de ces protéines est le facteur d'ADP-ribosylation ARF. ARF a été à l'origine identifié comme le cofacteur nécessaire à la réaction d'ADP-ribosylation de la sous-unité $\alpha$ de Gs provoquée par la toxine de Vibrio cholerae. Des travaux ultérieurs ont montré que les protéines ARF (on connaît actuellement six gènes $A R F$ chez les mammiferes et deux chez la levure) étaient en fait des protéines liant le GTP et le GDP, et $m / s n^{\circ} 4$, vol. 8, avril 92 relativement abondantes dans les cellules eucaryotes (environ $1 \%$ des protéines totales du cerveau). Cellesci sont myristylées et possèdent une activité GTP-ase intrinsèque très faible ou nulle. Quoique majoritairement cytosoliques, les formes membranaires d'ARF sont présentes sur les citernes de l'appareil de Golgi [38]. La première indication du rôle physiologique d'ARF a été la démonstration que, chez la levure Saccharomyces cerevisiae, la délétion d'un des deux gènes $A R F$ ( $A R F 1$ ) induit un phénotype proche de celui des mutants de YPT1 [38]. Une information importante vient d'être apportée par le groupe de Jim Rothman (NY, USA) qui a montré que ARF était une des protéines composant le manteau (coat) qui recouvre les vésicules de transport bourgeonnant de l'appareil de Golgi [39] (voir figure 1). Ces auteurs proposent que ARF sert à moduler le recrutement des protéines du manteau lors de la formation des vésicules de transport. ARF jouerait aussi un rôle dans le désassemblage (uncoating) des protéines du manteau. Des travaux non encore publiés indiquent, en outre, que ARF jouerait aussi un rôle dans d'autres étapes du transport intracellulaire, en particulier au cours de l'endocytose (Philippe Stahl, communication personnelle). Chez la levure, la protéine Sar1, proche structurellement d'ARF, est impliquée dans la formation des vésicules de transport quittant le réticulum endoplasmique [40, 41].

Plus inattendus sont les travaux encore préliminaires qui indiquent que les protéines $\mathrm{G}$ hétérotrimériques jouent aussi un rôle dans le transport intracellulaire. On savait déjà que le fluorure d'aluminium $\left(\mathrm{AlF}_{4}\right)$ inhibait, comme le GTP- $\gamma \mathrm{S}$, plusieurs étapes de transport in vitro. Ce composé active les protéines $G$ hétérotrimériques en mimant le troisième phosphate du GTP ( $\gamma$-phosphate) quand le GDP est présent dans le site nucléotidique. Plusieurs travaux viennent d'être publiés suggérant que le fluorure d'aluminium n'agit pas sur les protéines de la famille Sec4/Ypt1/rab ni sur ARF. Par ailleurs, il vient d'être montré que $\mathrm{G} \alpha_{i .3}$ est associée en partie aux citernes de l'appareil de Golgi et que sa surexpression inhibe le processus de sécrétion [42]. Ces résultats semblent indiquer que des protéines jusque-là impliquées dans des phénomènes de transduction de signaux au niveau de la membrane plasmique pourraient aussi régler les processus de transport intracellulaire.

\section{Conclusion}

L'identification des protéines de la famille Sec4/Ypt/rab a représenté, ces dernières années, une étape importante de l'étude des mécanismes moléculaires du transport des cellules eucaryotes. Même si l'on est encore loin de connaître en détail leur fonction et leur mode d'action, il semble maintenant acquis qu'elles jouent un rôle fondamental dans le contrôle du processus de transport vésiculaire. Les résultats obtenus récemment sur ARF et les protéines $G$ hétérotrimériques devraient ouvrir, dans un proche avenir, une nouvelle voie fascinante de recherche. On peut d'ores et déjà penser que trois GTP-ases différentes sont impliquées dans la régulation de chaque événement de transport.

La raison pour laquelle la cellule utilise un système apparemment aussi complexe pour contrôler le transport demeure pour l'instant un mystère. Ces résultats, associés à de nombreuses études portant sur les autres GTA-ases, confirment en tout cas que la cellule se sert des propriétés remarquables de ces protéines pour contrôler un grand nombre de fonctions essentielles

\section{Summary}

GTP-ases regulate vesicular transport in eukaryotic cells A number of small GTP-binding proteins have now been found associated with the membrane of vesicles and compartments involved in transport of macromolecules along the secretory and endocytic pathway. These proteins form a distinct branch of the ras superfamily, the Sec4/Ypt1/rab family. Increasing evidence indicates that they play a key regulatory role in many transport events. Very recently, other GTP-ases such as the ADP-ribosylation factor ARF and heterotrimeric $G$ proteins have also been involved in membrane trafficking. 